\title{
A new quantum model for Ohmic dissipation
}

\author{
Michael Wilkinson \\ Faculty of Mathematics and Computing, Open University, Walton Hall, \\ Milton Keynes MK7 6AA, UK
}

Received 16 March 2001, in final form 3 July 2001

Published 16 August 2001

Online at stacks.iop.org/JPhysCM/13/8087

\begin{abstract}
This paper discusses a model for dissipation in electrical conductors, in which a time-dependent flux $X(t)$ is threaded through a loop. The rate of dissipation is proportional to a constant $D_{E}$ characterizing the diffusion of single-particle energies, and Ohmic dissipation occurs when $D_{E} \propto \dot{X}^{2}$. The diffusion constant $D_{E}$ is investigated for a random-matrix model, and compared with the predictions of the Kubo-Greenwood formula. The results agree with the Kubo-Greenwood formula in a physically important regime where other random-matrix models do not show agreement. Numerical experiments also identified another regime of this model which exhibits Ohmic dissipation, with a conductance which does not agree with the Kubo-Greenwood formula.
\end{abstract}

\section{Introduction}

This paper discusses the validity of the Kubo formula [1], which is used to calculate the electrical conductance of metallic systems. This is a fundamental issue in the physics of metals, and is also representative of a general class of problems, in which a complex system responds to an external force by dissipating energy. The Kubo formula is a term used to cover a systematic approach to calculating linear response coefficients quantum mechanically, rather than a specific expression. The derivation uses perturbation theory, and it has been argued [2] that this is potentially a serious weakness. For example, the limit of validity of perturbation theory could be exceeded by unphysically small disturbances. The existing literature does not provide a satisfying answer to this question.

Previous papers [3, 4] have discussed circumstances in which the Kubo formula is shown to break down by the dissipation becoming non-Ohmic, in that the current ceases to be proportional to voltage. Reference [3] discusses the parameter regime where dissipation is determined by Landau-Zener transitions. This case is relevant to large, low-frequency perturbations of microscopic systems. Reference [4] discussed a case with wider implications. A random-matrix model was shown to exhibit markedly non-Ohmic response in a regime where a dimensionless parameter $\eta$ (which will be defined in section 2) is large. It is this regime which corresponds to typical physical situations (in which real conductors do show 
Ohmic behaviour). This observation was significant for two reasons. Firstly, random-matrix models have been so successful in predicting spectral fluctuations (on small energy scales) that it has become natural to assume that they will also correctly describe dynamical behaviour (correspondingly, at low frequencies). The results in [4] demonstrate a complete failure of the random-matrix model to exhibit physically realistic dynamical behaviour. Secondly, even if the model in [4] is unphysical, it does show that the Kubo formula is not universally valid.

This paper will describe a different random-matrix model, which is of interest for two reasons. Firstly, the model can give agreement with the Kubo formula in the regime where the earlier random-matrix model of reference [4] gives anomalous behaviour. This aspect is explained by an analytical (but non-rigorous) theory. Secondly, numerical experiments show that the model has a regime exhibiting Ohmic dissipation, but having a conductance which can differ by orders of magnitude from that predicted by the Kubo formula. This is a significant observation because it indicates that in some systems an experimentally observed Ohmic response might not be described correctly by the Kubo formula.

General arguments indicate that low-energy excitations of systems of interacting fermions typically behave as non-interacting quasi-particles. Also, the lifetime for interaction with phonons diverges as the temperature approaches zero. (These points are discussed clearly in [5]). At low temperatures the system may be assumed to consist of non-interacting fermions with single-particle Hamiltonian $\hat{H}(t)$. This is the standard model for charge transport in metals and semiconductors at low temperatures. In this paper I will be concerned exclusively with this independent-electron approximation. All of the effects which will be described could be manifest in the dynamics of electrons on short timescales. There is no reason to believe that they should be destroyed by residual interactions between electrons. For simplicity, I ignore the existence of electron spin.

The energies of single electrons undergo diffusion in response to a time-dependent perturbation. The rate of dissipation is proportional to the energy diffusion constant, $D_{E}$. This relationship between diffusion and dissipation is described in section 2, together with the version of the Kubo formula due to Greenwood [6], which is appropriate to the independentelectron model. Some earlier work discussing the range of validity of the Kubo formula is also discussed there.

Section 3 introduces the random-matrix model, and estimates its energy diffusion constant. Section 4 discusses the estimate for the rate of dissipation for this model according to the Kubo formula. It is shown that these estimates can agree both when the dimensionless parameter $\eta$ is large, and when it is small.

The arguments presented in this paper are not mathematically rigorous, and in most cases a precise formulation would be extremely difficult. Instead, the conclusions are tested by means of numerical experiments on the random-matrix model. These are discussed in section 5 . Agreement is found for parameter regimes within the validity of the theory given in section 3 . In a regime where this theory does not apply, I find Ohmic dissipation at a rate which does not agree with the Kubo-Greenwood formula.

In section 6 I will argue that the random-matrix model is closely analogous to the Anderson model for a particular choice of basis. The limit of weak disorder in the Anderson model corresponds to the regime in the random-matrix model for which the Kubo formula holds. The case in which the site energies are comparable to the hopping amplitudes corresponds to the crossover point in the present model, where the Kubo formula starts to fail.

I will use equals signs throughout this paper, but it will be clear from the context that many of the relations are approximate. 


\section{Summary of earlier work}

\subsection{Energy diffusion and dissipation}

The approach used here will be to determine the conductance by calculating the rate of dissipation. A simple formula can be used to calculate the rate of dissipation by a system of independent fermions at zero temperature. This relation is based upon the observation that the single-particle energies evolve diffusively: their change in energy $\Delta E(t)$ after the action of a perturbation over time $t$ satisfies

$$
\left\langle\Delta E^{2}(t)\right\rangle=2 D_{E} t .
$$

In the independent-electron model, this result leads to a simple but powerful picture of the mechanism for dissipation: diffusion of electrons from filled states below the Fermi energy into empty states above the Fermi energy results in an irreversible increase in the energy of the system. Provided that the temperature is sufficiently low that the variation of the diffusion constant and density of states in an interval of width $k_{\mathrm{B}} T$ around the Fermi energy may be neglected, the rate of dissipation is

$$
\frac{\mathrm{d} E}{\mathrm{~d} t}=\rho D_{E}
$$

where $\rho$ is the single-particle density of states. Both $\rho$ and $D_{E}$ are evaluated at the Fermi energy.

To the best of my knowledge, the first time that the existence of the energy diffusion relation (2.1) was explicitly remarked upon was in a paper by Ott [7]. Its use to compute the rate of dissipation using (2.2) was first described in [3, 8]. The formula (2.2) is a powerful tool for understanding dissipation because it shows that this problem is directly linked to energy diffusion, which in many circumstances is much easier to calculate.

The treatment of open quantum systems, where the number of particles is not fixed, can involve problematic assumptions about the role of the environment. It is possible to avoid having to represent electrons entering and leaving the sample by considering a system in the form of a loop, which is threaded by a magnetic flux, $X(t)$. The rate of change of the flux, $\dot{X}$, is the electromotive force acting around the loop. If the response of the loop is Ohmic, the current flowing around the loop is $I=\Sigma \dot{X}$ where $\Sigma$ is the conductance. The rate at which energy is dissipated is $\mathrm{d} E / \mathrm{d} t=I \dot{X}$. Comparison with (2.2) shows that in the case of Ohmic dissipation the energy diffusion constant is proportional to $\dot{X}^{2}$, and is related to the conductance as follows:

$$
D_{E}=\frac{\Sigma}{\rho} \dot{X}^{2}
$$

\subsection{The Kubo-Greenwood formula}

Early theories for electrical conductance were essentially classical, and for many years there was uncertainty about the form of a fully quantum mechanical theory. Kubo [1] gave expressions which can be recognized as a natural quantum mechanical extensions of classical linear response theory. These have become the accepted basis for quantum mechanical calculations of electrical conductance. These formulae are very general, but are somewhat formal, and it is not immediately clear how they should be evaluated.

Later Greenwood [6] gave a concrete formula for the electrical conductance for electrons treated as independent fermions moving in a static disordered potential. This formula is often referred to as the 'Kubo-Greenwood formula'. It expresses the conductance $\Sigma(\omega)$ in terms of 
matrix elements $j_{n m}$ of the current operator:

$$
\Sigma(\omega)=-\pi \hbar \sum_{n, m}\left|j_{n m}\right|^{2} \frac{\partial f}{\partial E}\left(E_{n}\right) \delta\left(\hbar \omega-E_{n}+E_{m}\right) .
$$

Here $\omega$ is the frequency of the electric field, and $f(E)$ is the single-particle occupation probability, which would normally be the Fermi-Dirac distribution function. It is the KuboGreenwood formula which is usually the basis for a concrete calculation of the residual conductance. The matrix elements are evaluated in the basis formed by the eigenstates $\left|\phi_{n}\right\rangle$ of the Hamiltonian. Spin degeneracy has been ignored in writing this expression.

If the electric field is introduced as a time-dependent flux in a loop, the matrix elements of the current operator appearing in (2.4) are matrix elements of the generalized force $\partial H / \partial X$ :

$$
j_{n m}=\left(\partial_{X} H\right)_{n m} \equiv\left\langle\phi_{n}\left|\frac{\partial \hat{H}}{\partial X}\right| \phi_{m}\right\rangle
$$

where the states $\left|\phi_{n}\right\rangle$ are the eigenstates of $\hat{H}(X)$. The Kubo-Greenwood formula may be expressed in terms of a statistic characterizing the variance $\sigma^{2}$ of these matrix elements, which is a function of the energies of the two states:

$\sigma^{2}(E, \Delta E) \equiv \frac{1}{\rho^{2}(E)}\left\langle\sum_{n m}\left|\left(\partial_{X} H\right)_{n m}\right|^{2} \delta\left(\Delta E-E_{n}+E_{m}\right) \delta\left(E-\frac{1}{2}\left(E_{n}+E_{m}\right)\right)\right\rangle$.

This function will be characterized by two parameters: $\sigma_{0}^{2}$ is its value at $\Delta E=0$, and its 'support' (the magnitude of the interval over which it differs significantly from zero) is $\Delta E_{\mathrm{c}}$. The angle brackets denote a suitable averaging procedure; in the case of the random-matrix model this is simply the ensemble average.

I will consider only the case where the flux $X(t)$ increases linearly with time, so the limit $\omega \rightarrow 0$ should be taken in order to obtain the DC conductance. The Kubo-Greenwood formula can be used to deduce an expression for the energy diffusion constant, $D_{E}$. In the low-temperature and low-frequency limits, equations (2.3) and (2.4) imply that

$$
D_{E}=\pi \hbar \rho\left(E_{\mathrm{F}}\right) \sigma^{2}\left(E_{\mathrm{F}}, 0\right) \dot{X}^{2} .
$$

If the Kubo-Greenwood formula is correct, the energy diffusion constant must be given by (2.7).

\subsection{Earlier work concerning the validity of the Kubo formula}

The derivation of the Kubo-Greenwood formula is essentially perturbative. The conditions for the validity of a quantum mechanical perturbation theory are potentially very stringent, because in macroscopic systems the separation of energy levels is extremely small. It is desirable to have an independent demonstration that the energy diffusion constant is correctly given by (2.7).

For systems which exhibit classical behaviour, this has been done. The energy diffusion constant was calculated for classical systems by Ott [7], who showed that it is related to the force-force correlation function:

$$
D_{E}=\frac{1}{2} \dot{X}^{2} \int_{-\infty}^{\infty} \mathrm{d} t\left\langle\frac{\partial H}{\partial X}(t) \frac{\partial H}{\partial X}(0)\right\rangle_{E}
$$

where the average $\langle\cdots\rangle_{E}$ is a microcanonical average at energy $E$. A semiclassical trace formula [9] relates the force-force correlation function to the matrix element variance:

$$
\sigma^{2}(E, \Delta E)=\frac{1}{2 \pi \hbar \rho} \int_{-\infty}^{\infty} \mathrm{d} t \exp (\mathrm{i} \Delta E t / \hbar)\left\langle\frac{\partial H}{\partial X}(t) \frac{\partial H}{\partial X}(0)\right\rangle_{E} .
$$


Combining (2.8) and (2.9) reproduces (2.7). This justifies the use of the Kubo-Greenwood formula in systems where the electrons behave semiclassically. It is however desirable to have an entirely quantum mechanical calculation of the energy diffusion constant.

Reference [4] discusses an attempt to do this. It models the adiabatic form of the Schrödinger equation by a stochastic differential equation. This model leads to equation (2.7) in the limit where

$$
\eta \equiv \hbar \rho \sigma_{0} \dot{X} / \Delta E_{\mathrm{c}} \ll 1 \text {. }
$$

When $\eta \gg 1$, the energy diffusion constant is predicted to be much smaller than would be obtained from (2.7), and non-Ohmic. This prediction was confirmed by numerical experiments on a random-matrix model, but it was also pointed out that the results are unphysical in the context of electrical conduction, because the parameter $\eta$ is large in typical experimental situations. One conclusion is that the model used in this study, a version of Wigner's banded random-matrix ensemble, has some pathological features. Another is that the KuboGreenwood formula is not universally applicable. The conditions under which the KuboGreenwood formula is applicable in purely quantum systems remain unclear.

Another case in which dissipation is not described by (2.7) occurs when Landau-Zener transitions are the mechanism for transitions between states. This was first proposed as a mechanism for dissipation by Hill and Wheeler [10], and was characterized using the diffusion-dissipation formula in [3]. The Landau-Zener mechanism would be observable only in microscopic systems subjected to large, low-frequency perturbations.

\section{Energy diffusion in a random-matrix model}

\subsection{A random-matrix model}

The Hamiltonian $\hat{H}(X)$ is an $N \times N$ matrix with elements $H_{i j}(X)$ :

$$
H_{i j}(X)=\cos \left(X+\phi_{i}\right) \delta_{i j}+\epsilon V_{i j}
$$

Here $\phi_{i}$ are random phases which are uniformly distributed on the interval $[0,2 \pi], \epsilon \ll 1$ is a coupling constant and $V_{i j}$ are elements of a real symmetric random matrix, with diagonal elements zero, and with elements in the upper triangle independently and identically distributed. The distribution of the $V_{i j}$ will be assumed to be Gaussian, with mean value zero and variance unity.

The eigenstates when $\epsilon=0$ are denoted $\left|\chi_{i}\right\rangle$. The wavefunction is expanded in this basis:

$$
|\psi(t)\rangle=\sum_{n=1}^{N} a_{n}(t) \exp \left[-\mathrm{i} \theta_{n}(t)\right]\left|\chi_{n}\right\rangle
$$

where $a_{n}(t)$ are coefficients determining the wavefunction. The phases $\theta_{n}$ are

$$
\theta_{n}(t)=\frac{1}{\hbar} \int_{0}^{t} \mathrm{~d} t^{\prime} \mathcal{E}_{n}\left(X\left(t^{\prime}\right)\right)
$$

where

$$
\mathcal{E}_{n}(X)=\cos \left(X+\phi_{n}\right)
$$

are the eigenvalues of the diagonal part of $\hat{H}(X)$. Note that the states $\left|\chi_{n}\right\rangle$ are independent of $X$ (in the case where the $\mathcal{E}_{n}(X)$ are degenerate, the $\left|\chi_{n}\right\rangle$ are defined to be independent of $X$ ). For simplicity, it will be assumed that the rate of change of $X(t)$ is constant:

$$
X(t)=\dot{X} t .
$$


If $X(t)$ is interpreted as the magnetic flux threaded through a loop, $\dot{X}$ is the electromotive force acting around the loop.

Substituting (3.2) into the Schrödinger equation, i $\hbar \partial_{t}|\psi\rangle=\hat{H}|\psi\rangle$, and multiplying by $\exp \left[i \theta_{n}(t)\right]\left\langle\chi_{n}\right|$, leads to the following equation of motion for the coefficient $a_{n}(t)$ :

$$
\dot{a}_{n}=-\frac{\mathrm{i} \epsilon}{\hbar} \sum_{m=1}^{N} V_{n m} \exp \left[\mathrm{i}\left(\theta_{n}-\theta_{m}\right)\right] a_{m} .
$$

This is the equation of motion which will be solved approximately to produce an estimate of the energy diffusion constant.

The density of states $\rho$ will be required. In the limit $\epsilon \rightarrow 0$, the spectrum lies in the interval $[-1,1]$ with density of states

$$
\rho(E)=\frac{N}{\pi} \frac{1}{\sqrt{1-E^{2}}} .
$$

\subsection{Energy diffusion constant}

Equation (3.6) will be treated perturbatively, in the following sense. The change $\Delta a_{n}$ of $a_{n}$ which occurs between zero time and time $\Delta t$ will be calculated by assuming that all of the $a_{m}$ may be regarded as constants over the interval $\Delta t$. The change in the coefficients is then

$$
\Delta a_{n}=-\frac{\mathrm{i} \epsilon}{\hbar} \sum_{m=1}^{N} V_{n m} a_{m} \int_{0}^{\Delta t} \mathrm{~d} t \exp \left[\mathrm{i}\left(\theta_{n}(t)-\theta_{m}(t)\right)\right] .
$$

The integrals are dominated by contributions from times where

$$
\frac{\mathrm{d}}{\mathrm{d} t}\left(\theta_{n}-\theta_{m}\right)=0=\mathcal{E}_{n}-\mathcal{E}_{m}
$$

corresponding to points where levels cross (in this model, the levels cross with slopes which are equal in magnitude but opposite in sign: $\mathrm{d} \mathcal{E}_{n} / \mathrm{d} X=-\mathrm{d} \mathcal{E}_{m} / \mathrm{d} X$ ). The time at which (3.9) is satisfied for levels $n$ and $m$ will be denoted $t_{n m}$. Also, define $\Theta_{n m}=\theta_{n}\left(t_{n m}\right)-\theta_{m}\left(t_{n m}\right)$. Then, if $\tau=t-t_{n m}$,

$$
\Delta \theta_{n m}(t) \equiv \theta_{n}(t)-\theta_{m}(t)=\Theta_{n m}+\frac{1}{2 \hbar}\left(\frac{\mathrm{d} \mathcal{E}_{n}}{\mathrm{~d} t}-\frac{\mathrm{d} \mathcal{E}_{m}}{\mathrm{~d} t}\right) \tau^{2}+\mathrm{O}\left(\tau^{3}\right) .
$$

The cubic term will be neglected. With this approximation, the integrals appearing in (3.8) are

$$
\begin{gathered}
I_{n m}=\int_{0}^{\Delta t} \mathrm{~d} t \exp \left[\mathrm{i}\left(\theta_{n}(t)-\theta_{m}(t)\right] \sim \exp \left[\mathrm{i} \Theta_{n m}\right] \int_{-\infty}^{\infty} \mathrm{d} \tau \exp \left[\mathrm{i} A \dot{X} \tau^{2} / \hbar\right]\right. \\
=\exp \left[-\mathrm{i}\left(\Theta_{n m} \pm \pi / 4\right)\right] \sqrt{\frac{\pi \hbar}{A \dot{X}}}
\end{gathered}
$$

where $A$ is the magnitude of the slope,

$$
A=\frac{\mathrm{d} \mathcal{E}}{\mathrm{d} X}=\sin \left(\cos ^{-1} E\right)=\sqrt{1-E^{2}}
$$

and where the sign in (3.11) is negative if $\mathrm{d} \mathcal{E}_{n} / \mathrm{d} X$ is positive. If there is no level crossing between $\mathcal{E}_{n}$ and $\mathcal{E}_{m}$ in time $\Delta t$, the integral $I_{n m}$ is negligible and is set equal to zero.

In first-order perturbation theory the expression for the change in the amplitudes $a_{n}$ may be written as

$$
\Delta a_{n}=\sum_{n=1}^{N} F_{n m} a_{m} \quad F_{n m}=-\frac{\mathrm{i} \epsilon}{\hbar} V_{n m} I_{n m}
$$


but this approximation does not conserve probability. An approximation which does conserve probability is obtained by writing

$$
a_{n}(t+\Delta t)=\sum_{m} U_{n m} a_{m}(t)
$$

where the unitary operator $\hat{U}=\left\{U_{n m}\right\}$ is obtained by exponentiating the operator $\hat{F}$ with matrix elements $F_{n m}$ :

$$
U_{n m}=\{\exp [\hat{F}]\}_{n m}=\delta_{n m}+F_{n m}+\frac{1}{2} \sum_{k} F_{n k} F_{k m}+\mathrm{O}\left(F^{3}\right) .
$$

The probability of reaching the state with index $n$ at time $t+\Delta t$ is then

$$
P_{n}(t+\Delta t)=\left\langle\left|a_{n}(t+\Delta t)\right|^{2}\right\rangle=\sum_{m} \sum_{m^{\prime}}\left\langle U_{n m} U_{n m^{\prime}}^{*} a_{m}(t) a_{m^{\prime}}(t)\right\rangle \sim \sum_{m}\left\langle\left|U_{n m}\right|^{2}\right\rangle P_{m}(t)
$$

where it has been assumed that the $a_{m}(t)$ are uncorrelated with each other, and with the elements $U_{n m}$. The transition probability from state $m$ to state $n$ is

$$
\begin{aligned}
\left\langle\left|U_{n m}\right|^{2}\right\rangle= & \left\langle\delta_{n m}+F_{n m}+F_{n m}^{*}+\left|F_{n m}\right|^{2}+\frac{1}{2} \sum_{k} F_{n k} F_{k m}+F_{n k}^{*} F_{k m}^{*}\right\rangle+\mathrm{O}\left(F^{3}\right) \\
& =\delta_{n m}+\left\langle\left|F_{n m}\right|^{2}\right\rangle+\operatorname{Re} \sum_{k}\left\langle F_{n k} F_{k m}\right\rangle+\mathrm{O}\left(F^{3}\right) \\
& =\delta_{n m}+\left\langle\left|F_{n m}\right|^{2}\right\rangle-\delta_{n m} \sum_{k}\left\langle\left|F_{n k}\right|^{2}\right\rangle+\mathrm{O}\left(F^{3}\right) .
\end{aligned}
$$

The cubic term is negligible. Subtracting $P_{n}(t)$ from $P_{n}(t+\delta t)$, and dividing by $\Delta t$, gives a rate equation for the probabilities:

$$
\frac{\mathrm{d} P_{n}}{\mathrm{~d} t}=\sum_{m} R_{n m}\left(P_{m}-P_{n}\right)
$$

where the rate constants $R_{n m}$ are

$$
R_{n m}=\frac{\left\langle\left|F_{n m}\right|^{2}\right\rangle}{\Delta t}
$$

When the levels with indices $n$ and $m$ cross, the mean squared magnitude of the factors $\epsilon V_{n m} I_{n m} / \hbar$ appearing in (3.13) is equal to $\pi \epsilon^{2} / A \dot{X} \hbar$. The mean value in the numerator of (3.19) is obtained by multiplying this by the expected number of level crossings in the interval $\Delta t$. This latter factor is equal to $r(E) \Delta t$, where

$$
r(E)=\frac{N}{\pi} \dot{X}
$$

is the rate at which any given level is crossed. The rate of transition to states with an opposite sign of the slope $\mathrm{d} \mathcal{E} / \mathrm{d} X$ is therefore

$$
R=\frac{N \epsilon^{2}}{\hbar \sqrt{1-E^{2}}}
$$

at energy $E$. Note that this expression is independent of the indices $n, m$, and of $\dot{X}$.

Now consider how to calculate the energy diffusion constant. When the unperturbed energies $\mathcal{E}_{n}(X)$ of two basis states cross, the slopes $\mathrm{d} \mathcal{E}_{n} / \mathrm{d} X$ are equal and opposite, with magnitude $A(E)$. An electron makes random transitions between these states. The change in the energy of an electron is

$$
\Delta E(t)=\dot{X} \int_{0}^{t} \mathrm{~d} t^{\prime} \frac{\mathrm{d} E}{\mathrm{~d} X}\left(t^{\prime}\right)=A(E) \dot{X} \int_{0}^{t} \mathrm{~d} t^{\prime} s\left(t^{\prime}\right)
$$


where $s(t)$ is the sign of the slope at time $t$, and where it is assumed that $\Delta E(t)$ is small compared with the support of the spectrum. The variance of $\Delta E(t)$ may be approximated by

$$
\left\langle\Delta E^{2}(t)\right\rangle \sim \dot{X}^{2} A^{2}(E) t \int_{-\infty}^{\infty} \mathrm{d} \tau\langle s(\tau) s(0)\rangle .
$$

(This expression is valid provided that $t$ is much greater than the support of the correlation function). Now

$$
C(\tau)=\langle s(\tau) s(0)\rangle=(+1) P_{\text {same }}+(-1) P_{\text {diff }}
$$

where $P_{\text {same }}$ is the probability that the slope has the same sign at time $\tau$ as at time 0 , and $P_{\text {diff }}$ is the probability that the signs differ. The number $n$ of sign changes in time $\tau$ has a Poisson distribution:

$$
P(n, \tau)=\frac{(R \tau)^{n}}{n !} \exp (-R \tau)
$$

so, for positive $\tau$,

$$
C(\tau)=\sum_{n=0}^{\infty}(-1)^{n} \frac{(R \tau)^{n}}{n !} \exp (-R \tau)=\exp (-2 R \tau) .
$$

The energy diffusion constant is therefore

$$
D_{E}=\frac{\left\langle\Delta E^{2}(t)\right\rangle}{2 t}=\frac{1}{2} \dot{X}^{2}\left(1-E^{2}\right) \int_{-\infty}^{\infty} \mathrm{d} \tau \exp (-2 R|\tau|) .
$$

Substituting the rate constant from (3.21):

$$
D_{E}=\frac{\left(1-E^{2}\right)^{3 / 2} \hbar}{2 N \epsilon^{2}} \dot{X}^{2} .
$$

Equation (3.28) is one of the central results of this paper. It is proportional to $\dot{X}^{2}$, indicating that the dissipation is Ohmic in this model.

This calculation made several significant assumptions. Firstly, it was assumed that the response is perturbative, in the sense that the transition probability in the basis which was used is small compared to unity, or equivalently that $\Delta a_{n}$ is small. This requires that the transition rate (3.21) multiplied by the time $\tau$ over which the integrals in (3.11) converge, namely $\tau \sim(\hbar / A \dot{X})^{1 / 2}$, is small:

$$
R \tau \sim \frac{N \epsilon^{2}}{A \sqrt{\hbar A \dot{X}}} \ll 1 .
$$

Secondly, it was assumed that the integral over the phase factor is dominated by the region close to the stationary phase point where the quadratic approximation in (3.10) is valid. This requires

$$
A \gg \dot{X} \hbar \text {. }
$$

Also, it was assumed that the energy levels are close to those of the unperturbed system, which requires

$$
\epsilon \ll 1 \text {. }
$$

Another assumption was that the time required to 'scatter' an electron from an increasing to a decreasing level, $R^{-1}$, is small compared to the timescale over which the energy of the level changes by an amount comparable to the width of the spectrum, $\tau_{1} \sim(A \dot{X})^{-1}$. This condition is

$$
R \tau_{1} \sim \frac{N \epsilon^{2}}{\hbar A^{2} \dot{X}} \ll 1
$$


The inequalities (3.29) to (3.32) are at least sufficient for the theory to apply. Note that (3.29), (3.30) and (3.31) taken together imply that

$$
\frac{N \epsilon^{2}}{A} \ll 1
$$

Numerical experiments (reported in section 5) show that this is a necessary condition for (3.28) to hold.

\section{Evaluation of the Kubo-Greenwood formula}

\subsection{Perturbative case}

This section discusses the calculation of the variance of matrix elements defined by (2.6). The estimate for this statistic depends upon the magnitude of the perturbation parameter $\epsilon$. In the case where $\epsilon \rho \ll 1$, the eigenstates $\left|\phi_{n}\right\rangle$ contain significant admixtures of only one or two of the basis states $\left|\chi_{n}\right\rangle$. In this case, the matrix elements are approximately

$$
\left(\partial_{X} H\right)_{n m}=\frac{\epsilon A V_{n m}}{\sqrt{A^{2} \delta X^{2}+\epsilon^{2}\left|V_{n m}\right|^{2}}}=\frac{\epsilon A V_{n m}}{\Delta E} .
$$

Here $\delta X=X-X_{0}$, where $X_{0}$ is the parameter value for which $\left|E_{n}-E_{m}\right|$ is smallest, and $A=\mathrm{d} \mathcal{E}_{n} / \mathrm{d} X$. This expression is valid when $E_{n} \sim E_{m}$; the matrix elements are negligible when this condition is not satisfied.

First consider the form of $\sigma^{2}(E, \Delta E)$ if all of the matrix elements $V_{n m}$ have the same magnitude, $V$. The average required to calculate (2.6) can be taken as an average over $X$, and can be written in the form

$$
\sigma^{2}(E, \Delta E)=\frac{1}{\rho \Delta X} \int_{0}^{\Delta X} \mathrm{~d} X\left|\partial_{X} H_{n m}\right|^{2} \delta\left(\Delta E-E_{n}+E_{m}\right)
$$

where $1 \gg \Delta X \gg 1 / N$ and $E_{m}$ is taken to be the closest energy to $E_{n}$ at any position $X$. Recalling that the frequency of crossings of the unperturbed levels is given by (3.20), and using (4.1):

$$
\begin{aligned}
\sigma^{2}(E, \Delta E) & =\frac{\epsilon^{2} A^{2} V^{2}}{\Delta E^{2}} \frac{\Delta N}{\rho \Delta X} \int \mathrm{d} X \delta\left(\Delta E(X)-E_{n}+E_{m}\right) \\
& =\frac{2 \epsilon^{2} A^{3} V^{2}}{\Delta E^{2}} \int_{-\infty}^{\infty} \mathrm{d} X \delta\left(X-X_{0}\right) \frac{1}{\left|\partial_{X} \Delta E\right|}
\end{aligned}
$$

where $\Delta N$ is the number of crossings in the interval $\Delta X$, and $X_{0}$ is one of the two parameter values at which $E_{n}-E_{m}=\Delta E$. Now

$$
\partial_{X} \Delta E=\partial_{X}\left(\sqrt{A^{2} X^{2}+\epsilon^{2} V^{2}}\right)=\frac{A \sqrt{\Delta E^{2}-\epsilon^{2} V^{2}}}{\Delta E} .
$$

This gives

$$
\sigma^{2}(E, \Delta E)=\frac{2 \epsilon^{2} A^{2} V^{2}}{\Delta E \sqrt{\Delta E^{2}-\epsilon^{2} V^{2}}} .
$$

This formula applies if all of the matrix elements $V$ are identical. In the case of interest, the elements $V$ are Gaussian distributed, with probability distribution $P(V)=\exp \left(-V^{2} / 2\right) / \sqrt{2 \pi}$. The variance of the matrix elements of the current is

$$
\sigma^{2}(E, \Delta E)=\frac{2 \epsilon^{2} A^{2}}{\Delta E^{2}} \int_{-\infty}^{\infty} \mathrm{d} V P(V) \frac{V^{2}}{\sqrt{1-\epsilon^{2} V^{2} / \Delta E^{2}}}=2 A^{2}\left(\frac{\epsilon^{2}}{\Delta E^{2}}\right) F(\Delta E / \epsilon)
$$


where

$$
F(x)=\frac{2}{\sqrt{2 \pi}} \int_{0}^{x} \mathrm{~d} y \frac{y^{2}}{\sqrt{1-y^{2} / x^{2}}} \exp \left(-y^{2}\right) .
$$

The function $F(x)$ has the following limits:

$$
\lim _{x \rightarrow \infty} F(x)=1 \quad \lim _{x \rightarrow 0} F(x) / x^{3}=\frac{\pi}{4} .
$$

\subsection{Non-perturbative case}

Next estimate $\sigma^{2}(E, \Delta E)$ for the case where $\epsilon$ is sufficiently large that the eigenstates $\left|\phi_{n}\right\rangle$ are admixtures of many of the $\left|\chi_{n}\right\rangle$ basis states. Write

$$
\left|\phi_{n}\right\rangle=\sum_{j=1}^{N} C_{j}^{(n)}\left|\chi_{j}\right\rangle
$$

It will be assumed that the coefficients $C_{j}^{(n)}$ are significant only when $E_{n} \sim \mathcal{E}_{j}$. In this case the required matrix elements may be approximated as follows:

$$
\left(\partial_{X} H\right)_{n m}=A\left(E_{n}\right) \sum_{i} s_{i} C_{i}^{(n)} C_{i}^{(m)}
$$

where $s_{i}$ is the sign of $\partial_{X} \mathcal{E}_{i}$. The variance of matrix elements will be estimated as follows:

\langle|$\left.\left.\left(\partial_{X} H\right)_{n m}\right)\left.\right|^{2}\right\rangle=A^{2} \sum_{i, j}\left\langle s_{i} s_{j} C_{i}^{(n)} C_{j}^{(n)} C_{i}^{(m)} C_{j}^{(m)}\right\rangle \sim A^{2} \sum_{i}\left\langle\left|C_{i}^{(n)}\right|^{2}\right\rangle\left\langle\left|C_{i}^{(m)}\right|^{2}\right\rangle$

where in writing the second equality it has been assumed that the $C_{i}^{(n)}$ are independent of each other, and of the signs $s_{i}$. Equation (4.11) is expressed in terms of the probability $P_{i}^{(n)}=\left\langle\left(C_{i}^{(n)}\right)^{2}\right\rangle$ that a particle in the $n$th eigenstate is in the $i$ th basis state. Perturbation theory indicates that the coefficients $C_{i}^{(n)}$ are approximately $\epsilon V_{n i} /\left(E_{n}-\mathcal{E}_{i}\right)$ when the denominator is sufficiently large that the magnitude of this coefficient is small. This is consistent with the local density of states taking a Lorentzian form

$$
P_{i}^{(n)}=\frac{\epsilon^{2}}{\alpha^{2}+\Delta E^{2}} \quad \Delta E=E_{n}-\mathcal{E}_{i}
$$

for some constant $\alpha$. A quite general argument supports the use of a Lorentzian function [11]. Normalizing so that the sum of the $P_{i}^{(n)}$ is unity determines the value of $\alpha$ :

$$
\alpha=\pi \epsilon^{2} \rho=\frac{N \epsilon^{2}}{A} \text {. }
$$

The statistic $\sigma^{2}(E, \Delta E)$ is now estimated using (4.11):

$\sigma^{2}(E, \Delta E)=\rho A^{2} \int_{-\infty}^{\infty} \mathrm{d} E^{\prime} \frac{1}{(\pi \epsilon \rho)^{2}+\left[\left(\Delta E-E^{\prime}\right) / \epsilon\right]^{2}} \frac{1}{(\pi \epsilon \rho)^{2}+\left(E^{\prime} / \epsilon\right)^{2}}$.

The function $\sigma^{2}$ is therefore also a Lorentzian:

$$
\sigma^{2}(E, \Delta E)=\frac{2 A^{2} \epsilon^{2}}{\Delta E^{2}+\left(2 \pi \rho \epsilon^{2}\right)^{2}}
$$

where $A$ and $\rho$ are evaluated at energy $E$. Equation (4.15) is valid provided $\rho \epsilon \gg 1$ and $\rho \epsilon^{2} \ll 1$. 


\subsection{Levels fully mixed}

The estimate (4.15) applies when the eigenstates are superpositions of many basis states, but the basis states with significant coefficients are drawn from those with a small range of unperturbed energies. When $\epsilon^{2} \rho \gg 1$, the eigenstates are admixtures of all of the basis states, and $P_{i}^{(n)} \sim 1 / N$. In this limit, all of the matrix elements of the current operator have the same magnitude. Assuming that $\sigma^{2}$ is independent of $E$ and $\Delta E$, its value can be deduced from a sum rule:

$$
\operatorname{tr}\left[\left(\frac{\partial \hat{H}}{\partial X}\right)^{2}\right]=\frac{1}{2} N=N^{2} \sigma^{2}(E, \Delta E)
$$

from which it follows that $\sigma^{2} \sim 1 / 2 N$.

\subsection{Comparison with energy diffusion formula}

Evaluation of the Kubo formula for the DC conductance requires an estimate of $\sigma^{2}\left(E_{\mathrm{F}}, \Delta E\right)$ in the limit $\Delta E \rightarrow 0$. There is often a difficulty with defining this limit: because of level repulsion effects, the function $\sigma^{2}(E, \Delta E)$ has an anomalous behaviour when $\Delta E \ll 1 / \rho$. In most circumstances the support $\Delta E_{\mathrm{c}}$ of this function greatly exceeds $1 / \rho$, and the relevant value of $\sigma^{2}$ is that obtained in the plateau where $1 / \rho \ll \Delta E \ll \Delta E_{\mathrm{c}}$.

In the first of the cases considered above, where $\epsilon N \ll 1$, the support of $\sigma^{2}(E, \Delta E)$ is $1 / \rho$, and there is no plateau region. The Kubo-Greenwood formula does not give a meaningful prediction for the DC conductance in this case, despite the fact that (3.28) can be applicable.

In the final case considered above, where $1 \gg \epsilon^{2} \gg 1 / N$, the estimate $\sigma_{0}^{2}=2 / N$ leads to an estimate for the energy diffusion constant which exceeds (3.28) by a factor $\sim N \epsilon^{2} / A^{4}$. However, the condition $\rho \epsilon^{2} \gg 1$ which defines this case is equivalent to requiring the converse of (3.33). The theory of section 3.2 need not apply. Numerical results on this case are discussed in section 5. They show that in this regime the diffusion constant is lower than that predicted by (3.28).

In the second case, where $\epsilon N \gg 1$ but $\epsilon^{2} N \ll 1$, the plateau value of $\sigma^{2}$ is

$$
\sigma_{0}^{2}=\frac{2 A^{2} \epsilon^{2}}{\left(2 \pi \rho \epsilon^{2}\right)^{2}}=\frac{A^{4}}{2 N^{2} \epsilon^{2}} .
$$

Substituting (3.7) and this expression into (2.7) leads to an expression which is identical to the prediction obtained in section 3 (equation (3.28)). The support in $\Delta E$ of the function $\sigma^{2}(E, \Delta E)$ is $\Delta E_{\mathrm{c}}=2 \pi \rho \epsilon^{2}$. The value of the parameter $\eta$ which played a crucial role in [4] is then

$$
\eta=\frac{\rho \sigma_{0} \hbar \dot{X}}{\Delta E_{\mathrm{c}}}=\frac{1}{2 \sqrt{2} \pi} \frac{A^{2}}{N \epsilon^{2}} \frac{\dot{X}}{\epsilon} .
$$

The conditions (3.29) to (3.33) allow $\eta$ to be either large or small. This random-matrix model need not, therefore, exhibit any anomalous behaviour when $\eta \gg 1$.

\section{Numerical experiments on the random-matrix model}

\subsection{Computations of energy diffusion}

A numerical estimate of the energy diffusion constant was obtained by solving the timedependent Schrödinger equation, using a fourth-order Runge-Kutta method. The program integrated the standard representation of the Schrödinger equation, in which the basis states are completely fixed, rather than the form (3.6) (in which the phase of the basis states evolves). 
A set of $\mathcal{N}$ initial states were integrated. These were eigenstates $\left|\phi_{m}(0)\right\rangle$ of the Hamiltonian at time $t=0$, chosen to be close to the centre of the spectrum. At regular intervals the state $|\psi(t)\rangle$ is projected into the basis formed by the instantaneous eigenstates, to determine coefficients $c_{n, m}$ defined by

$$
|\psi(t)\rangle=\sum_{n=1}^{N} c_{n, m}(t)\left|\phi_{n}(\dot{X} t)\right\rangle .
$$

The coefficients $c_{n, m}(t)$ are elements of the evolution operator expressed in the adiabatic basis They are used to calculate the variance of the displacement from the original state:

$$
\Delta(t) \equiv \frac{1}{\mathcal{N}} \sum_{m} \sum_{n=1}^{N}\left|c_{n, m}(t)\right|^{2}(n-m)^{2}
$$

where the sum over $m$ runs over $\mathcal{N}$ states close to the centre of the spectrum.

I used $\hbar=1$ in all calculations, and the number of propagated states $\mathcal{N}$ was never larger than $\mathcal{N}=N / 10$. The computations used 14-figure arithmetic, and the time step of the numerical integration was $\delta t=0.04$ or smaller.

The function $\Delta(t)$ defined in (5.2) typically grows quadratically for a short period, and then increases linearly until it starts to saturate, at a value which is approximately $N^{2} / 12$. An example is shown in figure 1 . The linear region of the plot was fitted to a straight line using a least-squares method. This fit is also shown for the example in figure 1. The slope $S$ of this linear fit is used to estimate the (numerical) energy diffusion constant, in terms of level index, $D_{n}^{\text {(num) }}=S / 2$. (The subscript $n$ indicates that this diffusion constant is defined through the second moment of the level index $n$, via the relation $\Delta(t)=\left\langle\Delta n^{2}\right\rangle \sim 2 D_{n} t$.) This is related to the energy diffusion constant introduced earlier by $D_{n}=\rho^{2} D_{E}$. The values of $D_{n}^{(\text {num })}$ are given in table 1 .

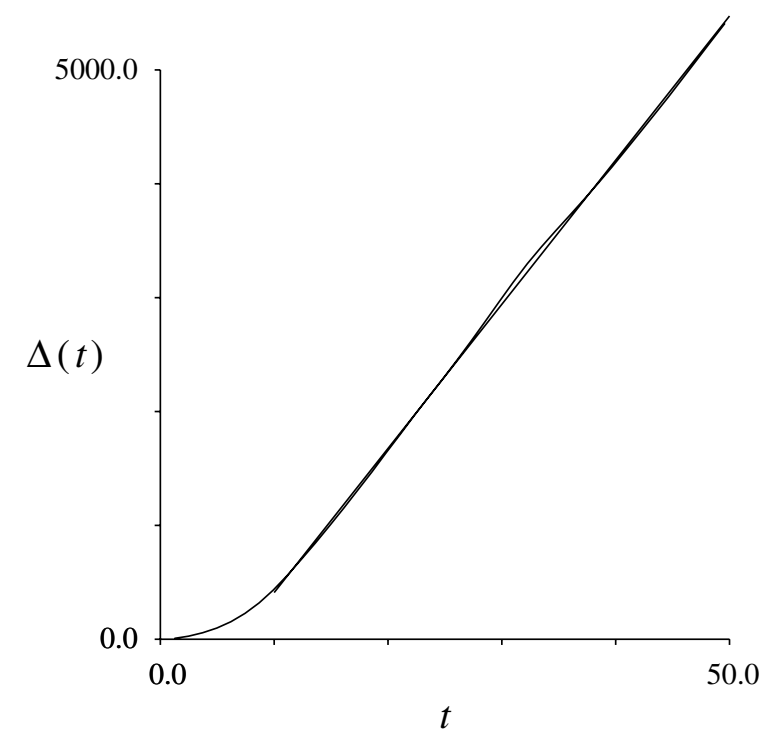

Figure 1. A plot of $\Delta(t)=\rho^{2}\left\langle\Delta E^{2}(t)\right\rangle$, showing the quadratic initial response followed by a diffusive spread level of occupation. A fit to the linear region is shown. The parameter values were $N=400, \epsilon=0.01, \dot{X}=0.02$. 
Table 1. Tabulation of the energy diffusion constant. The numerically determined value is $D_{n}^{\text {(num) }}$ and $f=D_{n}^{(\text {num })} / D_{n}^{(\text {th) }}$ is the ratio of this value to the theoretical prediction, equation (3.28). The results show that the theoretical prediction is accurate only when $x=N \epsilon^{2} \ll 1$, but that $D_{n}^{\text {(num) }}$ is approximately proportional to $\dot{X}^{2}$, indicating Ohmic response, for all of these cases.

\begin{tabular}{rllccl}
\hline$N$ & $\epsilon$ & $\dot{X}$ & $D_{n}^{\text {(num) }}$ & $x$ & $f$ \\
\hline 100 & 0.1 & 0.2 & 13.4 & 1 & 0.668 \\
160 & 0.1 & 0.2 & 18.1 & 1.6 & 0.564 \\
250 & 0.4 & 0.4 & 1.85 & 40 & 0.148 \\
250 & 0.4 & 0.2 & 0.532 & 40 & 0.168 \\
250 & 0.2 & 0.8 & 57.1 & 10 & 0.286 \\
250 & 0.2 & 0.4 & 14.8 & 10 & 0.296 \\
250 & 0.2 & 0.2 & 3.95 & 10 & 0.312 \\
250 & 0.2 & 0.1 & 1.04 & 10 & 0.333 \\
250 & 0.2 & 0.05 & 0.252 & 10 & 0.322 \\
250 & 0.2 & 0.025 & 0.063 & 10 & 0.320 \\
250 & 0.1 & 0.2 & 21.6 & 2.5 & 0.431 \\
250 & 0.1 & 0.1 & 6.75 & 2.5 & 0.538 \\
250 & 0.1 & 0.05 & 1.72 & 2.5 & 0.549 \\
250 & 0.1 & 0.025 & 0.433 & 2.5 & 0.554 \\
250 & 0.05 & 0.2 & 131 & 0.625 & 0.655 \\
250 & 0.05 & 0.1 & 37.1 & 0.625 & 0.742 \\
250 & 0.05 & 0.05 & 8.6 & 0.625 & 0.684 \\
250 & 0.025 & 0.05 & 40.9 & 0.156 & 0.818 \\
250 & 0.01 & 0.01 & 9.6 & 0.025 & 0.764 \\
250 & 0.01 & 0.005 & 2.75 & 0.025 & 0.882 \\
250 & 0.01 & 0.0025 & 0.75 & 0.025 & 0.954 \\
250 & 0.01 & 0.00125 & 0.193 & 0.025 & 0.986 \\
400 & 0.01 & 0.04 & 223 & 0.04 & 0.695 \\
400 & 0.01 & 0.02 & 63.9 & 0.04 & 0.788 \\
400 & 0.005 & 0.005 & 16.1 & 0.01 & 0.805 \\
400 & 0.005 & 0.0025 & 4.31 & 0.01 & 0.862 \\
400 & 0.005 & 0.00125 & 1.23 & 0.01 & 0.984 \\
500 & 0.4 & 3.2 & 170 & 80 & 0.105 \\
500 & 0.4 & 1.6 & 43.6 & 80 & 0.107 \\
500 & 0.4 & 0.8 & 10.9 & 80 & 0.107 \\
500 & 0.4 & 0.4 & 2.91 & 80 & 0.116 \\
500 & 0.4 & 0.2 & 0.790 & 80 & 0.125 \\
500 & 0.4 & 0.1 & 0.231 & 80 & 0.146 \\
500 & 0.01 & 0.01 & 21.5 & 0.05 & 0.858 \\
500 & 0.02 & 0.1 & 439 & 0.2 & 0.703 \\
1000 & 0.4 & 0.1 & 0.276 & 160 & 0.0871 \\
1000 & 0.5 & 0.5 & 3.32 & 250 & 0.0655 \\
\hline & 0.5 & 0.25 & 0.883 & 250 & 0.0697 \\
\hline & & & & &
\end{tabular}

Table 1 also compares the empirically determined diffusion constant with the theoretical estimates $D_{n}^{\text {(th) }}$ from (3.28). Setting $\hbar=1$, and also $A=1$ because I have selected states close to the centre of the spectrum, this is

$$
D_{n}^{(\text {th) }}=\frac{N \dot{X}^{2}}{2 \pi^{2} \epsilon^{2}} .
$$

The numerically determined diffusion constants were always lower than this theoretical prediction. This is partly because the theoretical diffusion constant is a function of energy, and the quoted theoretical values are the maximum ones. Also, the rate of growth of $\Delta(t)$ 
decreases as this statistic approaches its saturation value of $N^{2} / 12$. But it was clear that even if a correction were made for these effects, the diffusion constant would be lower than (5.3). The agreement with (5.3) is satisfactory when $N \epsilon^{2} \ll 1$ (i.e. when (3.33) is satisfied). When $N \epsilon^{2} \gg 1$ most of my numerical results were fairly consistent with the approximate expression

$$
D_{n}=\frac{K \sqrt{N}}{2 \pi^{2} \epsilon} \dot{X}^{2}
$$

where the value of the constant $K$ is approximately unity.

The ratio $f=D_{n}^{(\text {num })} / D_{n}^{(\text {th) }}$ is plotted as a function of $x=N \epsilon^{2}$ in figure 2, with straight lines corresponding to (5.3) and (5.4) (with $K=1$ ) superposed. If points corresponding to given values of $N$ and $\epsilon$ fall on top of each other, that is an indication that $D_{E} \propto \dot{X}^{2}$. The points do exhibit some vertical scatter, but the deviations from Ohmic response are usually small. For example, there are six data points at $N=250$ and $\epsilon=0.2$, with $\dot{X}$ changed by a factor of two between successive points. If the response of the system is Ohmic, the diffusion constant is expected to change by a factor of $4^{6-1}=1024$ between the largest and smallest values. The measured ratio was 906 , consistent with $D_{E} \propto \dot{X}^{1.96}$. Most of the data points cluster quite tightly about a single curve, but the scatter appears greater than that accounted for by the error in fitting the slope of $\Delta(t)$.

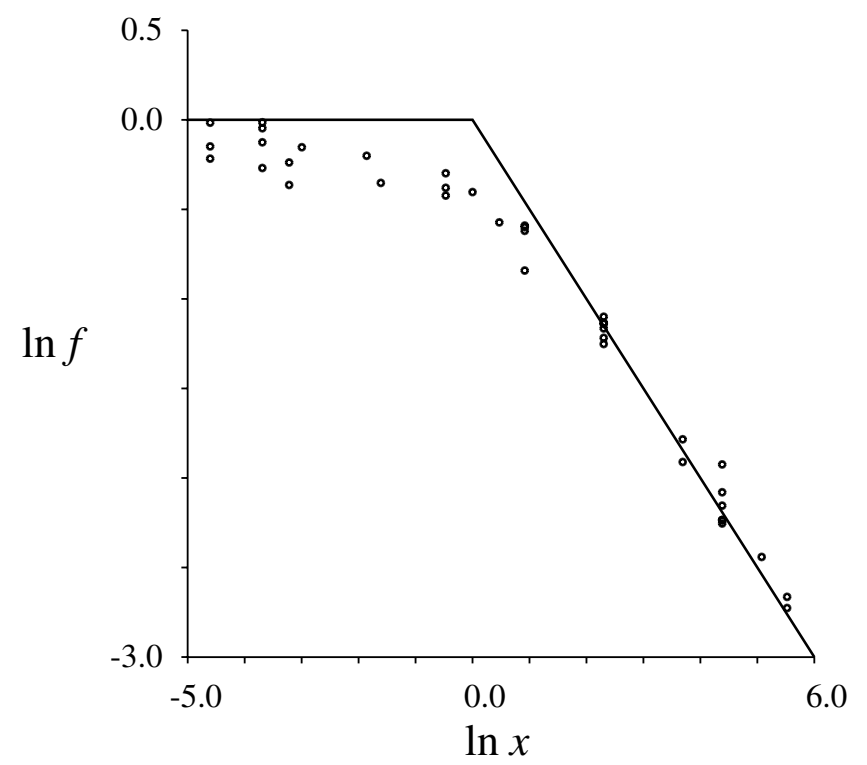

Figure 2. A plot of the ratio of the numerical diffusion constant to the theoretical expression given by (3.28). The ratio $f=D_{n}^{(\text {num) }} / D_{n}^{\text {(th) }}$ is close to unity when $x=N \epsilon^{2}$ is small, but when $x$ is large, it is approximated by $1 / \sqrt{x}$ (solid line).

\subsection{Computations of the matrix element variance}

The matrix elements (2.5) were obtained numerically by the following method. The Hamiltonian was diagonalized, and the diagonal matrix $\delta_{i j} \mathrm{~d} \mathcal{E}_{i}(X) / \mathrm{d} X$ was transformed into the basis formed by the eigenfunctions of $\hat{H}(X)$. The statistic $\sigma^{2}$ (defined by (2.6)) was estimated by taking elements corresponding to states with energies $E_{n}, E_{m}$ falling into bins 
satisfying $\frac{1}{2}\left(E_{n}+E_{m}\right)=E \pm \frac{1}{2} \delta E$ and $E_{n}-E_{m}=\Delta E \pm \frac{1}{2} \delta \Delta E^{\prime}$, and the results were averaged over $\mathcal{N}^{\prime}$ realizations of the random matrix.

The function $\sigma^{2}(0, \Delta E)$ is plotted in figure 3 for the case $N=400, \epsilon=0.01$. Here I used states from the centre of the spectrum, $E=0$, with a tolerance $\delta E=0.4$. The tolerance for $\Delta E$ was $\delta E^{\prime}=0.012$, and the number of realizations was $\mathcal{N}^{\prime}=25$. This case satisfies $N \epsilon \ll 1$ but $N \epsilon^{2} \ll 1$, so it corresponds to the regime considered in section 4.2 , and is expected to have a Lorentzian form. The fitted Lorentzian curve has parameters $\sigma_{0}^{2}=0.0263$ and $\Delta E_{\mathrm{c}}=0.0885$. These agree favourably with the theoretical predictions obtained from (4.15), namely $\sigma_{0}^{2}=1 / 32$ and $\Delta E_{\mathrm{c}}=0.08$.

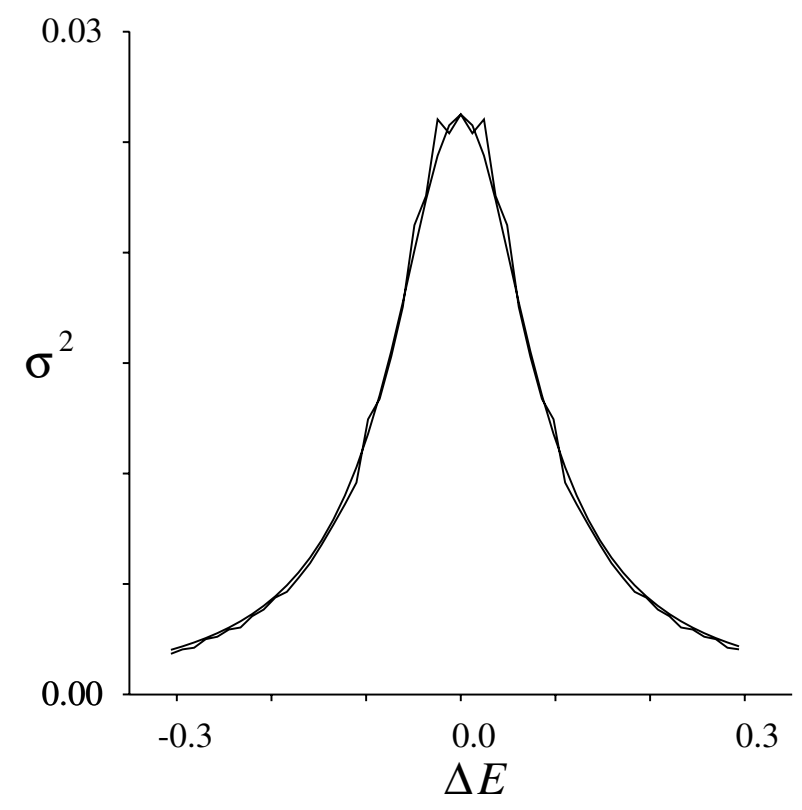

Figure 3. A plot of $\sigma^{2}(0, \Delta E)$, compared with a Lorentzian fit. The parameter values were $N=400, \epsilon=0.01$.

\section{Discussion of similarity to the Anderson model}

One motivation for the choice of the random-matrix model introduced in section 3 is that its matrix elements are similar to those of the Anderson Hamiltonian (tight-binding model with site disorder), expressed in the Bloch state basis. For simplicity, the discussion below is for the two-dimensional case, but the analogy extends to any dimension. In two dimensions states are believed to be always localized, but unless the disorder is strong the localization length is very large, and for practical purposes localization may be ignored, as is done in the following discussion.

The Hamiltonian for the Anderson model will be denoted by $\hat{H}(\Phi)=\hat{H}_{0}(\Phi)+\hat{V}$, where $\hat{H}_{0}(\Phi)$ is a tight-binding Hamiltonian on a finite $\left(N_{x} \times N_{y}\right)$ lattice with torus topology, enclosing a flux $\Phi$ through one loop, and $\hat{V}$ is the random fluctuation of the site energies. The dependence of the Hamiltonian on magnetic flux is incorporated by requiring that the matrix elements of the Hamiltonian $\hat{H}_{0}(\Phi)$ describing hopping from site $r=(i, j)$ to the 
neighbouring site $\boldsymbol{r}^{\prime}=(i+1, j)$ are of the form

$$
\left(H_{0}\right)_{r, r^{\prime}}=\exp \left[\mathrm{i} e \Phi(t) / N_{x} \hbar\right] .
$$

The Hamiltonian $\hat{H}_{0}(\Phi)$ is diagonal in the basis formed by the Bloch states $|\phi(\boldsymbol{k})\rangle$, with wavefunctions

$$
\langle\boldsymbol{r} \mid \phi(\boldsymbol{k})\rangle=\frac{\exp (\mathrm{i} \boldsymbol{k} \cdot \boldsymbol{r})}{\sqrt{N_{x} N_{y}}} .
$$

The wavevectors $k$ lie in the square $0 \leqslant k_{x}<2 \pi, 0 \leqslant k_{y}<2 \pi$, taking discrete values with density $N_{x} N_{y} /(2 \pi)^{2}$. The diagonal elements in the Bloch state basis are

$$
\left(H_{0}\right)_{k, k^{\prime}}=\delta_{k, k^{\prime}} \mathcal{E}\left(k_{x}-e \Phi / N_{x} \hbar, k_{y}\right)
$$

where $\mathcal{E}(\boldsymbol{k})=2 \cos \left(k_{x}\right)+2 \cos \left(k_{y}\right)$ is the dispersion relation for Bloch states with no magnetic flux. The diagonal elements are therefore periodic functions of the flux. This is a generalization of the form of the diagonal matrix elements of the model in section 3 . The matrix elements of the random potential $\hat{V}$ are

$$
V_{k, \boldsymbol{k}^{\prime}}=\left\langle\phi\left(\boldsymbol{k}^{\prime}\right)|\hat{V}| \phi(\boldsymbol{k})\right\rangle=\frac{1}{N_{x} N_{y}} \sum_{\boldsymbol{r}} V_{r} \exp \left[\mathrm{i}\left(\boldsymbol{k}-\boldsymbol{k}^{\prime}\right) \cdot \boldsymbol{r}\right]
$$

where $V_{r}$ is the random potential at lattice site $r$. The $V_{r}$ are assumed to have mean value zero and the variance is denoted by $\left\langle V^{2}\right\rangle$. The central-limit theorem implies that when $N=N_{x} N_{y}$ is large, the matrix elements in (6.4) have a Gaussian distribution. The correlation function of these matrix elements is

$$
\left\langle V_{\boldsymbol{k}_{1}, \boldsymbol{k}_{2}} V_{\boldsymbol{k}_{3}, \boldsymbol{k}_{4}}\right\rangle=\frac{\left\langle V^{2}\right\rangle}{N_{x} N_{y}} \delta_{\boldsymbol{k}_{1}+\boldsymbol{k}_{3}-\boldsymbol{k}_{2}-\boldsymbol{k}_{4}} .
$$

The matrix elements $V_{k, k^{\prime}}$ are similar to those of the random-matrix model introduced in section 3 , in that they all have the same variance. They differ by having correlations, which are not present in the random-matrix model.

Now consider which parameter regime of the random-matrix model corresponds to the Anderson model. The dimension of the random matrix corresponds to the number of sites in the Anderson model: $N=N_{x} N_{y}$. The parameter $X$ corresponds to the magnetic flux: $X=e \Phi / N_{x} \hbar$. Choosing $\epsilon$ so that the variance of the off-diagonal elements of the two models are identical requires

$$
N \epsilon^{2}=\left\langle V^{2}\right\rangle \text {. }
$$

This relation shows that the Anderson model with weak disorder corresponds to the regime discussed in section 4.2, for which the Kubo-Greenwood formula holds. The regime of moderate disorder corresponds to the crossover point, where the Kubo-Greenwood formula starts to fail. It would not be appropriate to deduce anything about the case of strong disorder, $\left\langle V^{2}\right\rangle \gg 1$, because the wavefunctions become well localized in that case.

\section{Conclusions}

The model introduced in this paper has two interesting dynamical properties. Firstly, in contrast to other random-matrix models (discussed in [4]), it does not show a catastrophic breakdown of Ohmic conduction when the parameter $\eta$ becomes large. This observation will help clarify which features of the Hamiltonian lead to pathological behaviour. Secondly, numerical experiments show that the model can display Ohmic dissipation with a conductance which is not given correctly by the Kubo-Greenwood formula. There can be a discrepancy of 
orders of magnitude between the two formulae. This demonstrates that the Kubo-Greenwood formula is not a universal expression for Ohmic conductance.

A similarity exists between the matrix elements of this model and those of the Anderson model. The correspondence between these models suggests that the latter should obey the Kubo-Greenwood formula for small disorder. When the disorder is comparable to the hopping matrix elements however, there may be a discrepancy between the conductivity predicted by the Kubo-Greenwood formula and the true value. The analogy between the models only holds good as far as the crossover regime, so the ratio between the true conductivity of the Anderson model and the Kubo-Greenwood value need not be large.

The Kubo-Greenwood formula is widely used as a theoretical basis for calculations of conductivity. There is a satisfying theoretical understanding of a vast range of experiments on electrical conduction, and it may appear surprising that a fundamental formula should be challenged. The following points should be made. Firstly, the Kubo-Greenwood formula can be represented as a quantum mechanical version of a classical linear response theory, which is beyond doubt (one approach to justifying this was outlined in section 2.3). If the electrons behave semiclassically the Kubo-Greenwood formula must be valid, because it is a quantum representation of the classical theory. In many situations the electron motion is well described by considering motion along classical trajectories, and the only places at which quantum mechanics enters are via the Pauli exclusion principle and simple interference effects. Secondly, it should be pointed out that in experimental applications of the Kubo-Greenwood formula, parameters such as scattering rates are fitted to the data, because disorder in the sample is not independently measured. In this sense the Kubo-Greenwood formula is therefore not fully tested experimentally.

There is room for doubt that the Kubo-Greenwood formula gives an accurate evaluation of the conductance when the electron motion is purely quantum. In this paper a discrepancy has been dramatically demonstrated for a random-matrix model, and the results suggest that a similar but less marked discrepancy could exist in the physically realistic Anderson model.

\section{References}

[1] Kubo R 1956 Can. J. Phys. 34 1274-7

[2] van Kampen N G 1971 Acta Phys. Norveg. 5 279-84

[3] Wilkinson M 1988 J. Phys. A: Math. Gen. 21 4021-37

[4] Wilkinson M and Austin E J 1995 J. Phys. A: Math. Gen. 28 2277-96

[5] Ashcroft N W and Mermin N D 1976 Solid State Physics (Philadelphia, PA: Saunders College)

[6] Greenwood D A 1958 Proc. Phys. Soc. A 68 585-96

[7] Ott E 1979 Phys. Rev. Lett. 42 1628-31

[8] Wilkinson M 1990 J. Phys. A: Math. Gen. 23 3603-11

[9] Wilkinson M 1987 J. Phys. A: Math. Gen. 20 2415-23

[10] Hill R L and Wheeler J H 1952 Phys. Rev. 89 1102-45

[11] Wilkinson M and Walker P N 1995 J. Phys. A: Math. Gen. 28 6143-60 